\title{
Electron backscattering diffraction and X-ray diffraction studies of interface relationships in $\mathrm{Sr}_{3} \mathrm{Ru}_{2} \mathrm{O}_{7} / \mathrm{Sr}_{2} \mathrm{RuO}_{4}$ eutectic crystals
}

\author{
R. Ciancio ${ }^{\mathrm{a}, \mathrm{b}, \mathrm{c}, *}$, H. Pettersson $^{\mathrm{b}}$, R. Fittipaldi ${ }^{\mathrm{a}}$, A. Kalabukhov ${ }^{\mathrm{d}}$, P. Orgiani ${ }^{\mathrm{e}}$, \\ A. Vecchione ${ }^{\mathrm{a}}$, Y. Maeno ${ }^{\mathrm{f}}$, S. Pace ${ }^{\mathrm{a}}$, E. Olsson ${ }^{\mathrm{b}}$ \\ a CNR-SPIN and Department of Physics “E.R. Caianiello”, University of Salerno, I-84081 Baronissi (SA), Italy \\ ${ }^{\mathrm{b}}$ Department of Applied Physics, Microscopy and Microanalysis, Chalmers University of Technology, SE-41296 GÄoteborg, Sweden \\ c CNR-IOM, TASC Laboratory, Area Science Park - Basovizza, I-34149 Trieste, Italy \\ d Department of Microtechnology and Nanoscience, Chalmers University of Technology, SE-41296 GÄoteborg, Sweden \\ e CNR-SPIN, I-84081 Baronissi (SA), Italy \\ ${ }^{\mathrm{f}}$ Department of Physics, Graduate School of Science, Kyoto University, Kyoto 606-8502, Japan
}

\section{A R T I C L E I N F O}

\section{Article history:}

Received 1 January 2010

Accepted 10 March 2010

\section{Keywords:}

EBSD

FEG-SEM

X-ray diffraction

Eutectic structure

\begin{abstract}
A B S T R A C T
$\mathrm{Sr}_{3} \mathrm{Ru}_{2} \mathrm{O}_{7} / \mathrm{Sr}_{2} \mathrm{RuO}_{4}$ eutectic system is investigated by electron backscattering diffraction (EBSD) and Xray diffraction (XRD). The eutectic growth enables the solidification of the two phases in an ordered lamellar pattern extending along the growth direction, namely the $b$-axis direction. The eutectic material thus provides in the $a-c$ plane two distinct interfaces having different microstructures with respect to the growth direction. Our analysis shows that, across the inplane $c$-axis direction (characterized by a poor lattice matching), the $b$-axis orientation is not constant at the individual interfaces, showing an orientation spread of about $5^{\circ}$. However, across the in-plane $a$-axis direction (characterized by a good lattice matching), the $b$-axis orientation does not change within a few tenths of degree $\left(\right.$ about $0.25^{\circ}$ ). Such information at nanoscale is also verified on a macroscopic level by standard XRD investigation.
\end{abstract}

(C) 2010 Elsevier Ltd. All rights reserved.

\section{Introduction}

Perovskite oxides display a wide variety of technologically important phenomena such as high temperature superconductivity, ferroelectricity, ferromagnetism, colossal magnetoresistance and metallic conductivity. Among them, a great role is held by Ruddlesden-Popper (R-P) series of $\mathrm{Sr}_{n+1} \mathrm{Ru}_{n} \mathrm{O}_{3 n+1}$ strontium ruthenates, nature's engineered layered systems with varying functionalities as the number of Ru-O layers per unit cell, $n$, increases (Cao et al., 1999). Besides the unconventional spin triplet superconductor $\mathrm{Sr}_{2} \mathrm{RuO}_{4}(n=1)$ (Mackenzie and Maeno, 2003), the R-P series also includes the $n=2$ bilayered member, $\mathrm{Sr}_{3} \mathrm{Ru}_{2} \mathrm{O}_{7}$ (Perry et al., 2004), which is a metamagnetic normal metal with a quantum critical point, the nearly ferromagnetic $\mathrm{Sr}_{4} \mathrm{Ru}_{3} \mathrm{O}_{10}(n=3)$ (Crawford et al., 2002; Cao et al., 2003) and the itinerant ferromagnet $\mathrm{SrRuO}_{3}$ $(n=\infty)$ (Eom et al., 1992; Allen et al., 1996; Mazin and Singh, 1997; Klein et al., 1996a,b; Cao et al., 1997; Kim et al., 2003). Because of the high structural and chemical similarities between the members of the series, new heterostructures can be obtained by merging together two homologue compounds in a unique com-

\footnotetext{
* Corresponding author at: CNR-SPIN and Department of Physics "E.R. Caianiello", University of Salerno, I-84081 Baronissi (SA), Italy.

E-mail address: ciancio@tasc.infm.it (R. Ciancio).
}

posite structure. A novel route to engineer normal metal/ $\mathrm{Sr}_{2} \mathrm{RuO}_{4}$ natural junctions has been successfully pursued by growing the superconducting $\mathrm{Sr}_{2} \mathrm{RuO}_{4}$ in combination with the normal metal $\mathrm{Sr}_{3} \mathrm{Ru}_{2} \mathrm{O}_{7}$ in $\mathrm{Sr}_{3} \mathrm{Ru}_{2} \mathrm{O}_{7} / \mathrm{Sr}_{2} \mathrm{RuO}_{4}$ eutectic crystals (Fittipaldi et al., 2004, 2008; Ciancio et al., 2009a). Extensive transmission electron microscopy (TEM) and high angle annular dark field (HAADF) scanning TEM (STEM) studies have shown that the eutectic material, in which the two component oxides are arranged in an ordered lamellar pattern, provides natural junctions characterised by two kinds of $\mathrm{Sr}_{3} \mathrm{Ru}_{2} \mathrm{O}_{7} / \mathrm{Sr}_{2} \mathrm{RuO}_{4}$ interface planes having different nanostructures depending on their orientation with respect to the growth direction of the crystal (Ciancio et al., 2009b). Interfaces parallel to the a-direction appear sharp and defect free with a quasi perfect atomic fit between the two phases. However, a profound wavy habit is observed at the interfaces parallel to the $c$-direction as a result of the high differences between the $c$-axis parameters of the two phases. Interfaces parallel to the growth direction of the crystal are identified as the best candidates to explore the order parameter of $\mathrm{Sr}_{2} \mathrm{RuO}_{4}$ through tunnelling experiments. Although TEM is indispensable to explore the atomic structure of the interfaces, Electron backscattered diffraction (EBSD) is a powerful tool to elucidate the local crystallographic orientation relationships on a micrometric scale thus enabling a visualisation and determination of the microstructural properties between the current paths, the crystallographic orientation and the fabrication parameters. 
Crystallographic orientation maps can be obtained by stepping the electron beam across an area of the sample. The results are given in orientation maps where a colour is associated to the crystallographic axis parallel to the sample surface normal. Up to now, only a few experiments have been reported on EBSD analysis performed on directionally solidified samples (Fujimoto et al., 2000; Koblischka-Veneva et al., 2002; Ramìrez-Rico et al., 2008; Grossin et al., 2006), mostly because of the serious difficulties concerning the Kikuchi pattern (KP) quality, which eventually does not allow the crystallographic mapping of the different phases. In this work, EBSD investigation of $\mathrm{Sr}_{3} \mathrm{Ru}_{2} \mathrm{O}_{7} / \mathrm{Sr}_{2} \mathrm{RuO}_{4}$ eutectic crystals is reported. In particular, a technique to obtain high-quality crystallographic phase maps and the factors influencing the measurement are also discussed. Through the maps, the crystallographic orientation relationships of the $\mathrm{Sr}_{3} \mathrm{Ru}_{2} \mathrm{O}_{7} / \mathrm{Sr}_{2} \mathrm{RuO}_{4}$ interfaces in both the crystallographic planes have been clearly presented along with quantitative information. To statistically explore the crystallographic orientation of the two phases on a macroscopic scale, structural properties of eutectic crystals have been also investigated by X-ray diffraction (XRD) technique.

\section{Experimental details}

$\mathrm{Sr}_{3} \mathrm{Ru}_{2} \mathrm{O}_{7} / \mathrm{Sr}_{2} \mathrm{RuO}_{4}$ eutectic crystals have been grown by flux feeding floating-zone (FFFZ) technique with Ru self-flux, using a commercial image furnace equipped with double-elliptical mirrors and two $2.0 \mathrm{~kW}$ halogen lamps (Fittipaldi et al., 2004). The FFFZ technique (Lichtenberg et al., 1992) consists of melting the bottom end of a feed rod suspended from above and connecting the molten zone to a seed held from below. Microstructural investigation of the crystals was carried out using a Leo Ultra 55 field emission gun (FEG) scanning electron microscope (SEM) with attached Oxford INCA Energy 300 energy-dispersive X-ray spectroscopy (EDS) system. The EBSD experiments were performed using the same microscope operated in the secondary electron mode, to locate interesting areas. Since EBSD is very sensitive to crystalline perfection, a well prepared sample is a prerequisite to obtaining a good diffraction pattern. For this purpose, flat surfaces in both planes perpendicular $(a-c)$ and parallel $(a-b)$ to the growth direction of the eutectic crystals have been prepared by polishing with alumina from $3 \mu \mathrm{m}$ to $0.3 \mu \mathrm{m}$ and subsequently with $20 \mathrm{~nm}$ colloidal silica. Using this method, a surface layer, approximately 2-3 $\mu \mathrm{m}$ in thickness, was removed. During EBSD analysis, the sample was tilted at $70^{\circ}$ to the horizontal to optimize both the contrast in the diffraction pattern and the fraction of electrons scattered

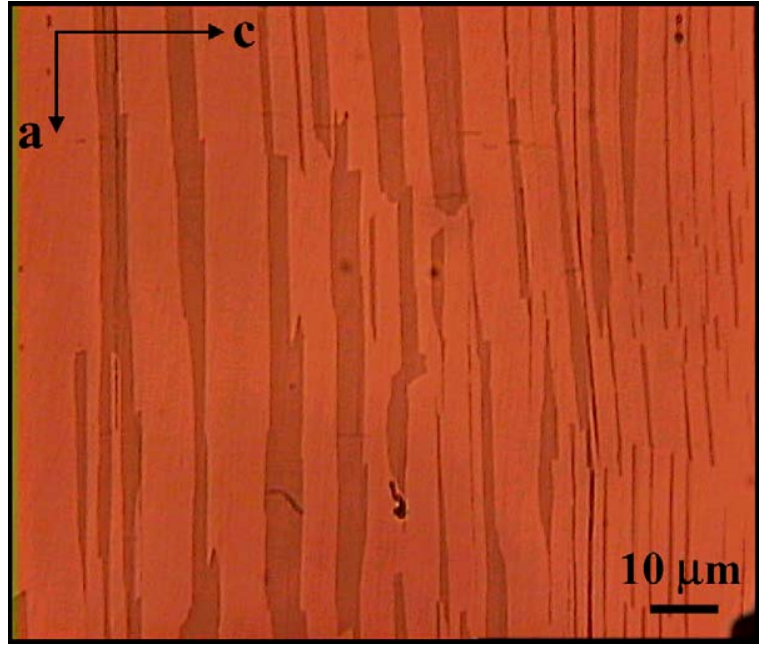

Fig. 1. Overview of the typical $\mathrm{Sr}_{3} \mathrm{Ru}_{2} \mathrm{O}_{7}-\mathrm{Sr}_{2} \mathrm{RuO}_{4}$ lamellar pattern of the eutectic solidification in the $a-c$ plane taken by polarized light optical microscopy: lamellae of $\mathrm{Sr}_{2} \mathrm{RuO}_{4}$ (dark contrast stripes) are embedded in the $\mathrm{Sr}_{3} \mathrm{Ru}_{2} \mathrm{O}_{7}$ region (bright contrast).

from the sample. An aperture size of $120 \mu \mathrm{m}$ and a working distance of $10 \mathrm{~mm}$ were used, with an electron high tension of $20 \mathrm{kV}$ and probe current of $10 \mathrm{nA}$. The phosphor screen was inserted at $175 \mathrm{~mm}$ from the sample. An EBSD detector and software from HKL technology were used. X-ray diffraction analysis was performed by using a Philips X'Pert-XRD analytic diffractometer equipped with a four-circle cradle. A Cu K $\alpha 1(\lambda=1.5406 \AA)$ source was used at $40 \mathrm{kV}$ and $40 \mathrm{~mA}$.

\section{Results and discussion}

\subsection{EBSD analysis}

In the $\mathrm{Sr}_{3} \mathrm{Ru}_{2} \mathrm{O}_{7} / \mathrm{Sr}_{2} \mathrm{RuO}_{4}$ eutectic crystals, the $\mathrm{Sr}_{3} \mathrm{Ru}_{2} \mathrm{O}_{7}$ and $\mathrm{Sr}_{2} \mathrm{RuO}_{4}$ phases solidify along the $b$ direction (growth direction) in an ordered pattern lamellar, as shown in the image of Fig. 1 taken by polarized light optical microscope. As further confirmed by energy dispersive $\mathrm{X}$-ray microanalysis, the dark contrast stripes are $\mathrm{Sr}_{2} \mathrm{RuO}_{4}$ lamellae embedded in the brighter contrast $\mathrm{Sr}_{3} \mathrm{Ru}_{2} \mathrm{O}_{7}$ matrix.

To perform EBSD experiments, the crystallographic data of the $\mathrm{Sr}_{3} \mathrm{Ru}_{2} \mathrm{O}_{7}$ and $\mathrm{Sr}_{2} \mathrm{RuO}_{4}$ phases were entered into the customized
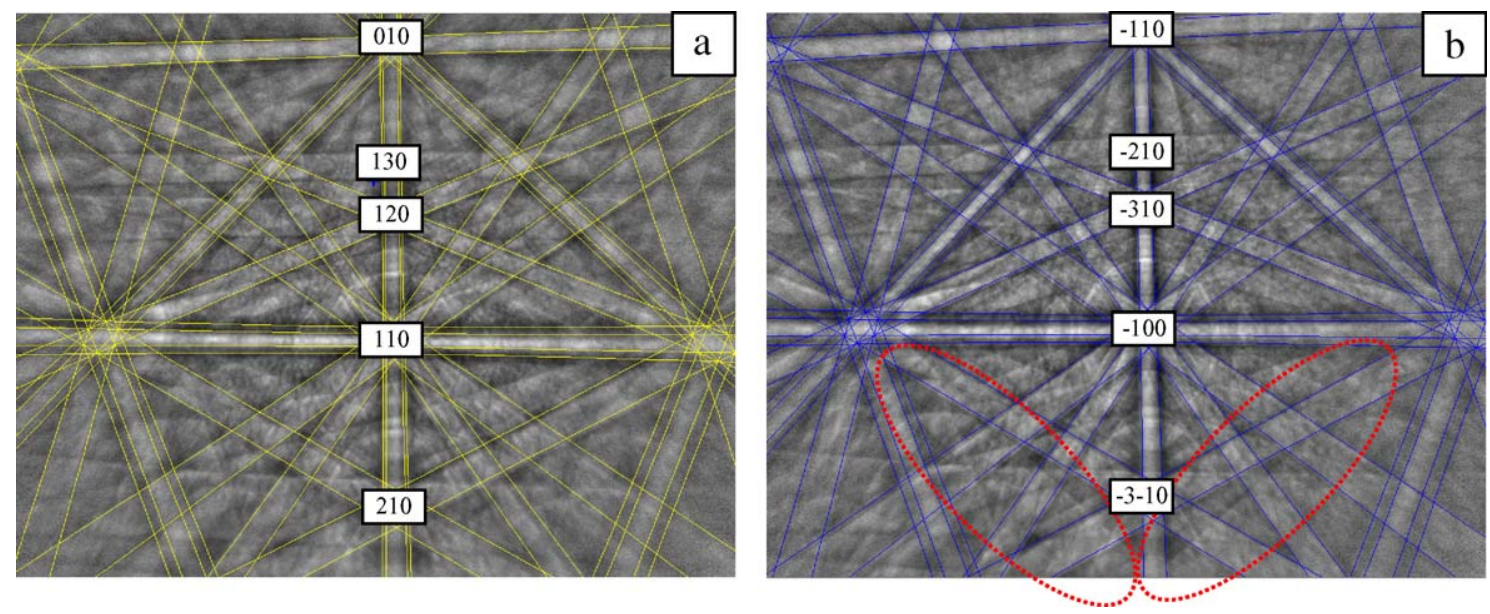

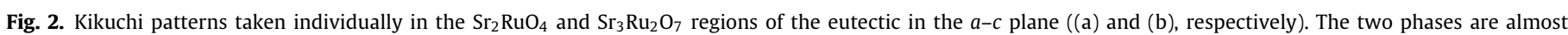
indistinguishable except for two supplementary weak bands detected in the Kikuchi pattern of the $\mathrm{Sr}_{3} \mathrm{Ru}_{2} \mathrm{O}_{7}$, as indicated by the dotted-ellipses. 

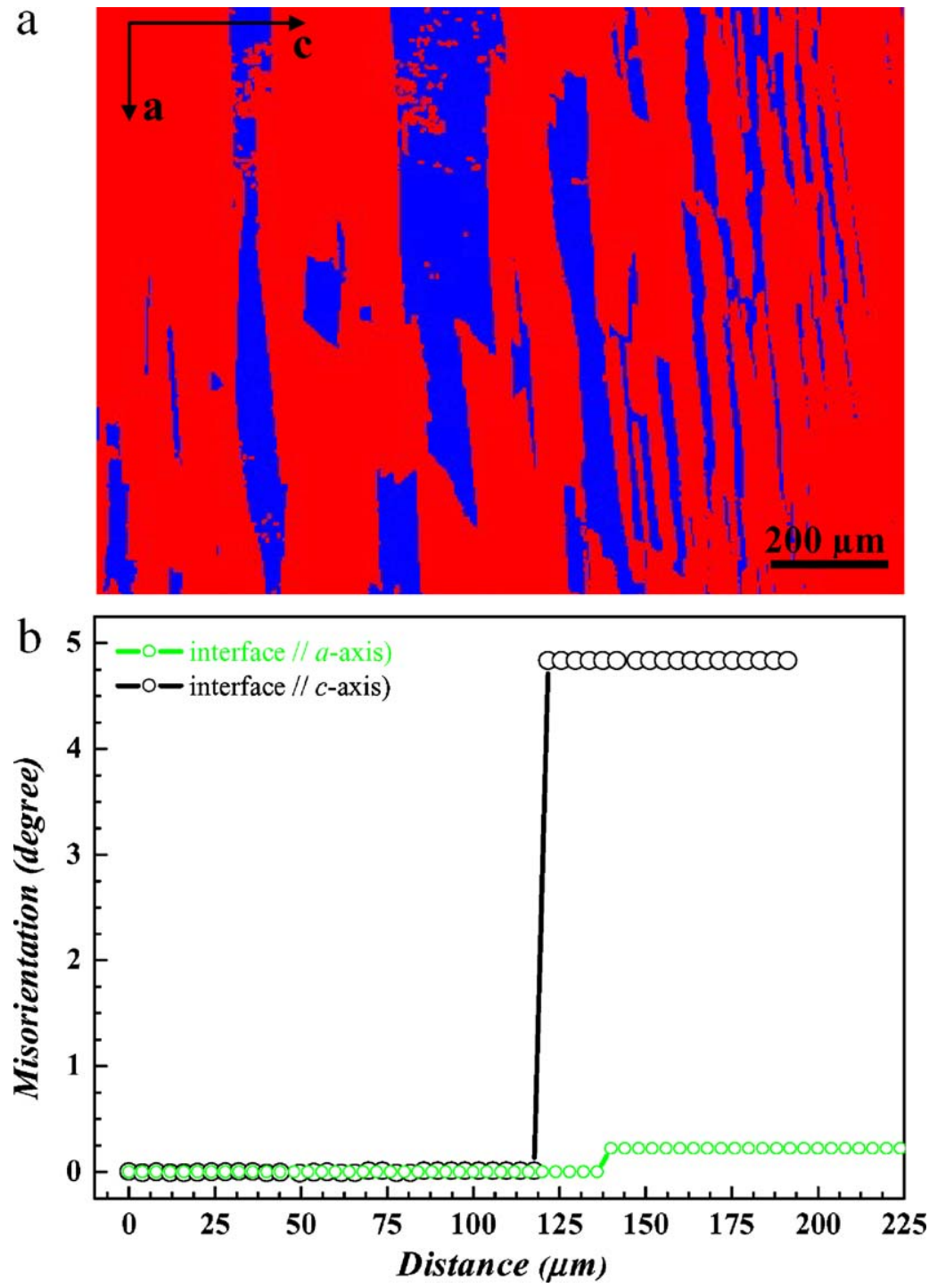

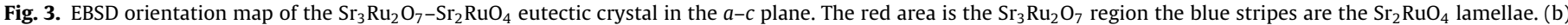

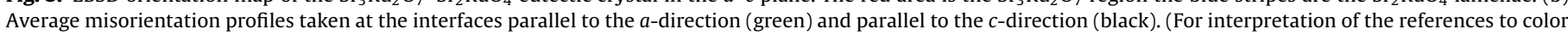
in this figure legend, the reader is referred to the web version of this article.)

HKL Channel 5 software database for the phase identification. Although $\mathrm{Sr}_{3} \mathrm{Ru}_{2} \mathrm{O}_{7}$ belongs to the orthorhombic space group $B b c b$ (Huang et al., 1998; Shaked et al., 2000) a tetragonal crystal structure with $I 4 / \mathrm{mmm}$ space-group symmetry was chosen for both $\mathrm{Sr}_{2} \mathrm{RuO}_{4}$ and $\mathrm{Sr}_{3} \mathrm{Ru}_{2} \mathrm{O}_{7}$. As also reported in the case of EBSD investigation of $\mathrm{YBa}_{2} \mathrm{Cu}_{3} \mathrm{O}_{7-x}$ (Grossin et al., 2006), this is found to yield to a considerable improvement in the phase detection and map quality since the EBSD system hardly discriminates between the slightly different $a$ and $b$ axes when an orthorhombic crystal structure is chosen. The HKL software automatically suggests solutions ranked by lowest 'mean angular deviation' (MAD) as an index of 'goodness of fit'. MAD values smaller than 1 are benchmark for accurate solutions. Each solution selected in the present case was the highest ranked solution and typically exhibited a MAD between 0.2 and 0.6. $\mathrm{KP}$ of the $\mathrm{Sr}_{2} \mathrm{RuO}_{4}$ and $\mathrm{Sr}_{3} \mathrm{Ru}_{2} \mathrm{O}_{7}$ regions in the $a-c$ plane are displayed in Fig. 2a and b, along with the corresponding indexing. The KP of the two phases only differ for two additional bands of weaker intensity revealed in the $\mathrm{KP}$ of $\mathrm{Sr}_{3} \mathrm{Ru}_{2} \mathrm{O}_{7}$, as enlightened by the red dotted ellipses.

Because of the high similarities between the crystallographic parameters of the two phases (as a matter of fact the two phases only differ for the $c$-axis parameters), the signal-to-noise ratio dur- ing the analysis has been progressively refined by subtracting the background at about camera saturation limit and by increasing the number of the indexed spots. Both conditions give highest signal gain for the detection of the Kikuchi bands. The KP were automatically indexed by the data collection unit, then analysed in the data analysis software package to determine the spatial distribution of the $\mathrm{Sr}_{2} \mathrm{RuO}_{4}$ and $\mathrm{Sr}_{3} \mathrm{Ru}_{2} \mathrm{O}_{7}$. Representative EBSD phase map of the $a-c$ plane of the $\mathrm{Sr}_{3} \mathrm{Ru}_{2} \mathrm{O}_{7} / \mathrm{Sr}_{2} \mathrm{RuO}_{4}$ eutectic crystal is shown in Fig. 3a. The optimal fit was obtained by stepping the beam across the surface with a step size of $4 \mu \mathrm{m}$. The map shows an extremely ordered phase separation with a preponderance of $\mathrm{Sr}_{3} \mathrm{Ru}_{2} \mathrm{O}_{7}$ phase (red colour pixels) in which the $\mathrm{Sr}_{2} \mathrm{RuO}_{4}$ lamellae (blue stripes) are embedded. Small inclusions of $\mathrm{Sr}_{3} \mathrm{Ru}_{2} \mathrm{O}_{7}$ are also detected within the $\mathrm{Sr}_{2} \mathrm{RuO}_{4}$ regions, because of the high similarity between the KP of the two phases in this projection. The phase distribution, quantified in $60 \%$ of $\mathrm{Sr}_{3} \mathrm{Ru}_{2} \mathrm{O}_{7}$ and $40 \%$ of $\mathrm{Sr}_{2} \mathrm{RuO}_{4}$, is in agreement with $\mathrm{X}$-ray diffraction and Rietveld analysis performed on powdered crystals. From the EBSD orientation map, information about the orientation relationships between the two phases at the $\mathrm{Sr}_{3} \mathrm{Ru}_{2} \mathrm{O}_{7} / \mathrm{Sr}_{2} \mathrm{RuO}_{4}$ interfaces across the two in-plane crystallographic direction has been obtained. Line scan profiles have been drawn across the interfaces parallel to the $a$-direction and paral- 

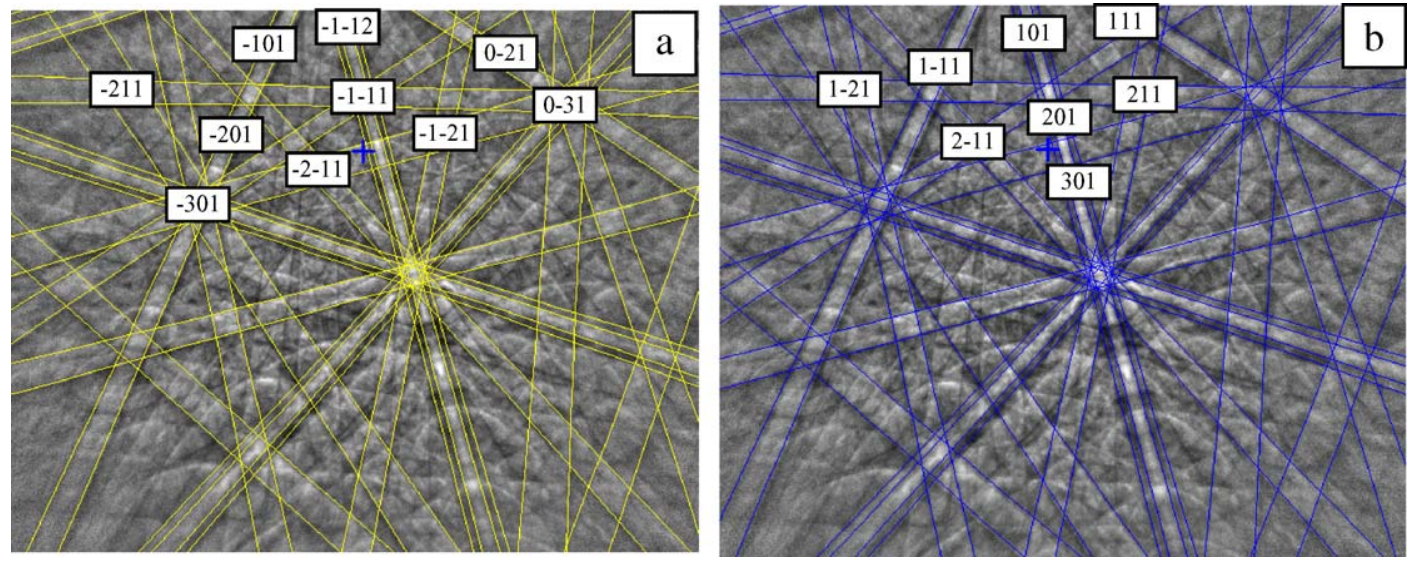

Fig. 4. Kikuchi patterns in the $a-b$ plane of the $\mathrm{Sr}_{2} \mathrm{RuO}_{4}$ (a) and $\mathrm{Sr}_{3} \mathrm{Ru}_{2} \mathrm{O}_{7}$ (b). The patterns are identical in this crystallographic plane.

lel to the $c$-direction and the misorientation profiles calculated by the software. The calculated average misorientation profiles across the two interfacial regions are shown in Fig. 3b. A misfit of nearly $0.25^{\circ}$ is usually recorded moving across the interfaces parallel to the $a$-direction while a misorientation angle of about $5^{\circ}$ is measured across the interface parallel to the c-direction. Taking into account the Euler angle definition given by Bunge (1985), the misorientation fluctuation is found by the software as being a variation of only the $\Phi$ Euler angle, which corresponds to a misorientation of $5^{\circ}$ between the $b$-axis of the two phases. Interfaces along the $a$-direction appear less misaligned, thus resulting as the best candidate to perform tunnel experiments. These results are in agreement with our investigations by TEM and HAADF/STEM analysis (Ciancio et al., 2009b).

Unlike the $a-c$ plane interfaces, in the $a-b$ plane the achievement of high quality EBSD orientation maps is heavily thwarted because of the high similarities of the in-plane axis parameters. Fig. 4 shows the KP taken in the $a-b$ plane of the $\mathrm{Sr}_{2} \mathrm{RuO}_{4}$ (a) and $\mathrm{Sr}_{3} \mathrm{Ru}_{2} \mathrm{O}_{7}$ (b) phase which are indistinguishable in this projection.

Identification of the two crystallographic phases by EBSD analysis can be achieved by using a step-size of $2 \mu \mathrm{m}$. A SEM image of the $a-b$ plane taken by secondary electrons is shown in Fig. 5a. In contrast to the well ordered lamellar pattern in the $a-c$ plane, a much more disordered distribution is observed in the $a-b$ planes. As shown in the orientation map of Fig. 5b, taken over the scanned image, $\mathrm{Sr}_{2} \mathrm{RuO}_{4}$ regions appear as isolated islands (blue regions) embedded in the $\mathrm{Sr}_{3} \mathrm{Ru}_{2} \mathrm{O}_{7}$ matrix (red colour areas) with irregular interfaces. As for EBSD investigation of the $a-c$ plane, also here inclusions of $\mathrm{Sr}_{3} \mathrm{Ru}_{2} \mathrm{O}_{7}$ are detected in the $\mathrm{Sr}_{2} \mathrm{RuO}_{4}$ areas, as a result of the EBSD software misindexing which even more easily in this projection can yield to cell misattribution. Nevertheless, the presence of such misindexed regions represent a minor feature of the EBSD overall investigation.

\section{2. $X R D$ diffraction}

The crystallographic properties of eutectic crystal have been also investigated on a macroscopic scale. The angles $\omega, 2 \theta$, and $\Phi$ were used according to the standard definition in XRD: $\omega$ represents the angle between the sample surface and incident beam, $2 \theta$ the angle between the incident and diffracted beams, $\Phi$ the rotation angle around the sample surface normal. XRD measurements have been performed on a millimeter-sized crystal, cut along the $b$-axis parameter. $\theta-2 \theta$ spectra only shows the $(010)$ peaks, thus confirming the orientation of the crystal along the [0 $\left.\begin{array}{lll}0 & 0\end{array}\right]$ crystallographic direction. In order to investigate the crystal quality along the a and $c$-axis, rocking curve measurements ( $\omega$-scan) were performed
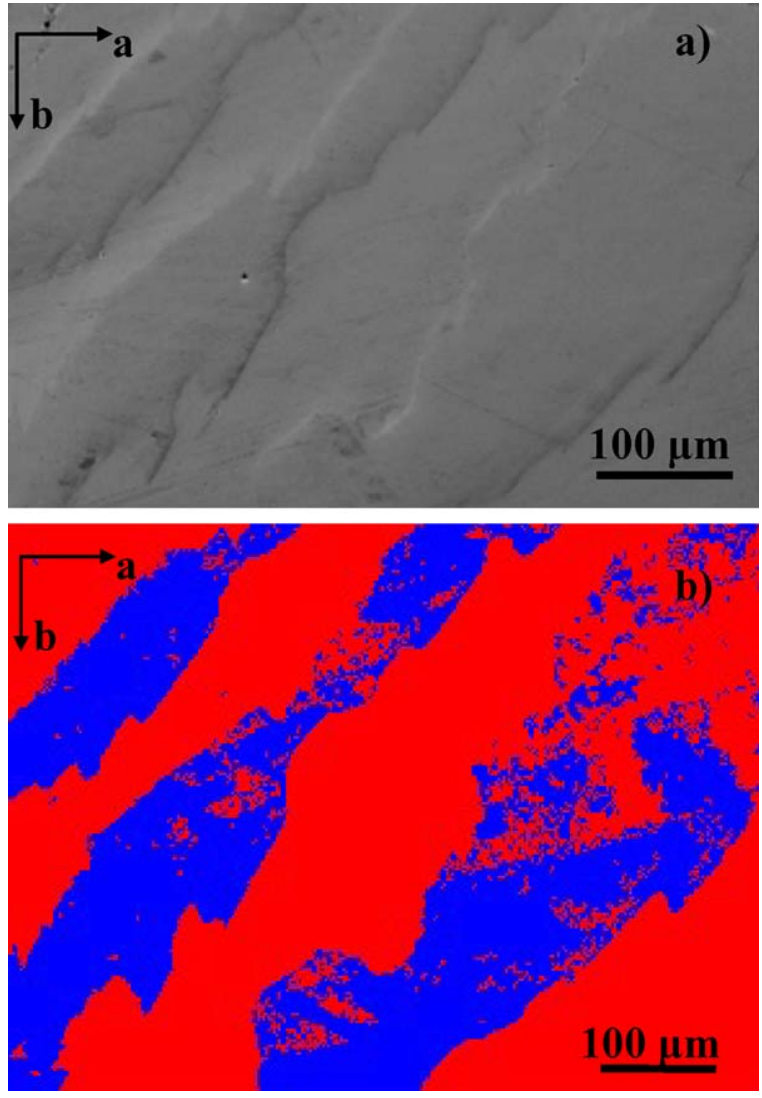

Fig. 5. (a) $\mathrm{SEM}$ micrograph of the $\mathrm{Sr}_{3} \mathrm{Ru}_{2} \mathrm{O}_{7}-\mathrm{Sr}_{2} \mathrm{RuO}_{4}$ eutectic crystal in the $a-b$ plane. (b) EBSD orientation map in the $a-b$ plane of a $\mathrm{Sr}_{3} \mathrm{Ru}_{2} \mathrm{O}_{7}-\mathrm{Sr}_{2} \mathrm{RuO}_{4}$ sample. The red area is the $\mathrm{Sr}_{3} \mathrm{Ru}_{2} \mathrm{O}_{7}$ region and the blue stripes are the $\mathrm{Sr}_{2} \mathrm{RuO}_{4}$ regions. (For interpretation of the references to color in this figure legend, the reader is referred to the web version of this article.)

(Fittipaldi et al., 2009). In particular, 2 $\theta-\omega$ maps around [0 20 ] reflection of $\mathrm{Sr}_{3} \mathrm{Ru}_{2} \mathrm{O}_{7} / \mathrm{Sr}_{2} \mathrm{RuO}_{4}$ eutectic were obtained performing the scan at $\Phi=0^{\circ}$ and $\Phi=90^{\circ}$. These two geometrical configuration correspond to the case in which the $a$-axis and the $c$-axis are aligned with the X-ray beam, respectively (see Fig. 6b, zoomed-out of the dash-edged region of Fig. 6b).

The $\omega$-scans provide information about the overall alignment of the $\mathrm{Sr}_{3} \mathrm{Ru}_{2} \mathrm{O}_{7} / \mathrm{Sr}_{2} \mathrm{RuO}_{4}$ unit cells parallel with respect to the two in-plane axes. By comparing the two spectra it is evident that a narrower rocking curve of the $b$-axis direction is observed across the well-matched $a$-axis interfaces (Fig. 6c), with respect 

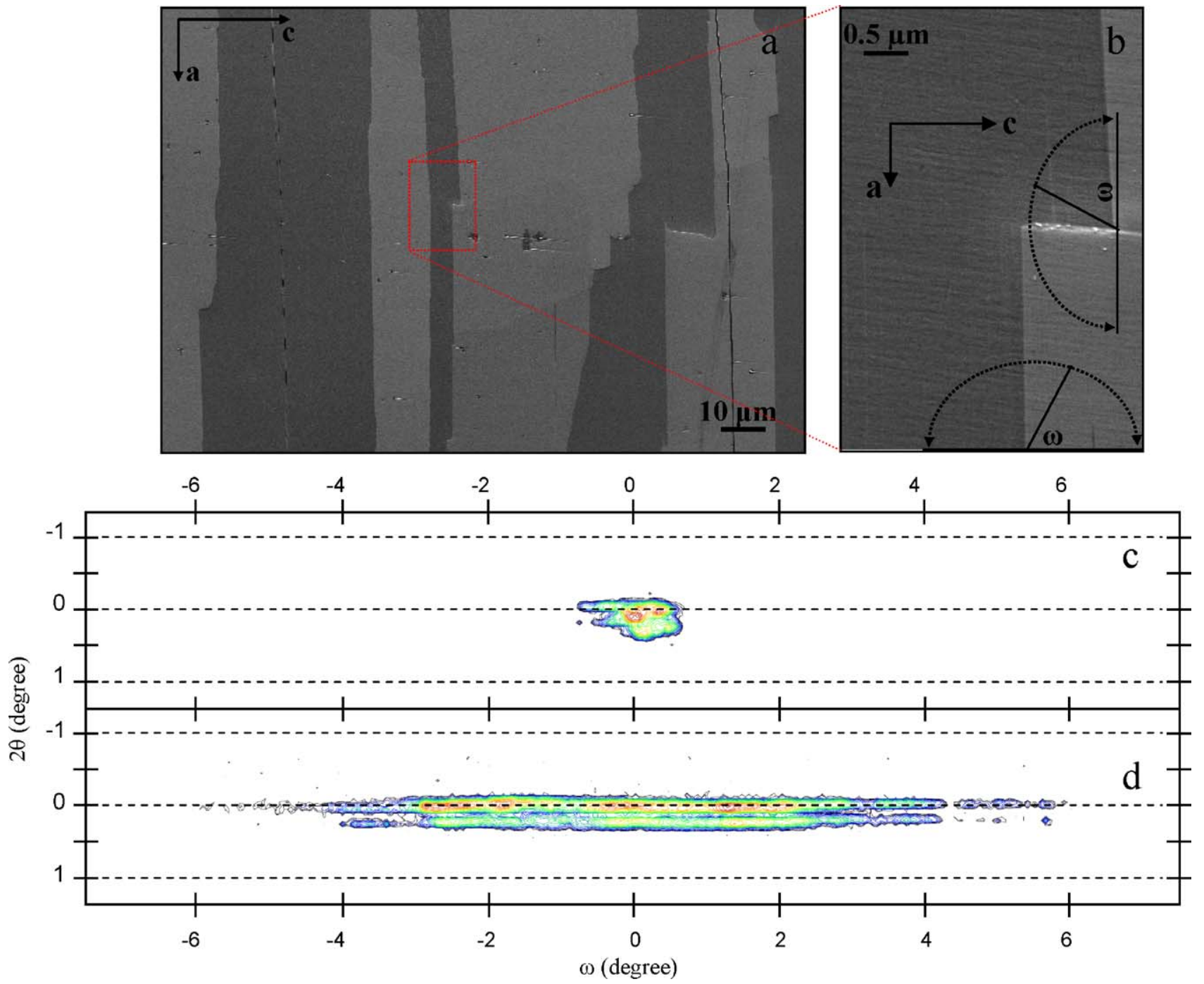

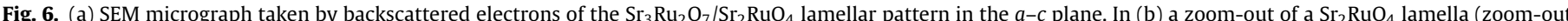

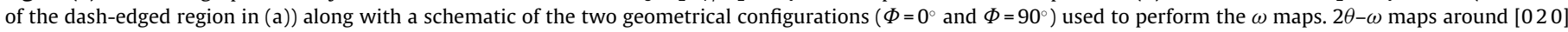
reflection of $\mathrm{Sr}_{2} \mathrm{RuO}_{4}-\mathrm{Sr}_{3} \mathrm{Ru}_{2} \mathrm{O}_{7}$ eutectic obtained performing the scan at $\Phi=0^{\circ}$ (c) and $\Phi=90^{\circ}$ (d).

to those obtained across the poorly-matched $c$-axis interfaces (Fig. 6d). In other words, the better interfacial matching of the $\mathrm{Sr}_{3} \mathrm{Ru}_{2} \mathrm{O}_{7} / \mathrm{Sr}_{2} \mathrm{RuO}_{4}$ unit cells along the $a$-axis direction provides a better alignment also among the $b$ crystallographic axes. However, in the case of the interfaces along the in-plane $c$-axis direction, which have a wavy habit when observed at the TEM (Ciancio et al., 2009b), the worse interfacial matching turns into a overall misalignment of the $b$ axes of unit cells at interface, confirmed by a larger dispersion in the $\omega$-scan.

\section{Conclusion}

In our work we explored the macroscopic texture and interface orientation relationships in $\mathrm{Sr}_{3} \mathrm{Ru}_{2} \mathrm{O}_{7} / \mathrm{Sr}_{2} \mathrm{RuO}_{4}$ eutectics grown by flux feeding floating-zone technique, by analysis of orientation maps obtained by EBSD experiments. Our analysis shows that the two $\mathrm{Sr}_{3} \mathrm{Ru}_{2} \mathrm{O}_{7}$ and $\mathrm{Sr}_{2} \mathrm{RuO}_{4}$ phases coherently grow along their $[010] \mathrm{Sr}_{3} \mathrm{Ru}_{2} \mathrm{O}_{7} / /[010] \mathrm{Sr}_{2} \mathrm{RuO}_{4}$ direction. Due to the strong difference between the $c$-axis parameters of the two phases, a large misalignment (about $5^{\circ}$ ) between the facing cells of the $b$ crystallographic direction is measured. However, at the interfaces parallel to the $a$-direction, because of the better matching between

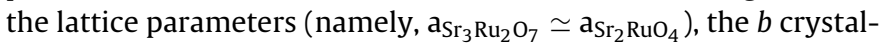

lographic axes result well aligned (only a very small misalignement of about $0.25^{\circ}$, between the $b$ crystallographic axes, is measured). XRD investigation on macroscopic areas of eutectic crystals confirm the two qualitatively different interfacial properties. Based on our investigation, further proofs that interfaces parallel to the $a$-direction are the best candidate for the study of proximity effect at the $\mathrm{Sr}_{3} \mathrm{Ru}_{2} \mathrm{O}_{7} / \mathrm{Sr}_{2} \mathrm{RuO}_{4}$ interfaces as well as for the fabrication of tunnel junctions are provided.

\section{Acknowledgedments}

The authors thanks A. Kvist and J. Weidow for the assistance in the EBSD measurements and for valuable scientific discussions. The SSF program OXIDE, a VR grant, and the Swedish National Graduate School of Material Science are thankfully acknowledged for financially supporting this work. Moreover, R.C. acknowledges the Swedish Institute, the Blanceflor Boncompagni-Lodovisi Foundation, the "Angelo della Riccia" Foundation, and the ESF activity Thin Films for Novel Oxide Devices (THIOX) and the European Community's Seventh Framework Programme 2007-2011 under NFFA Grant Agreement No. 212348 for partially supporting her research activity. 


\section{References}

Allen, P.B., Berger, H., Chauvet, O., Forro, L., Jarlborg, T., Junod, A., Revaz, B., Santi, G., 1996. Phys. Rev. B 53, 4393.

Bunge, H.J., 1985. An Introduction to Modern Texture Analysis. Academic Press Inc., UK.

Cao, G., McCall, S., Shepard, M., Crow, J.E., Guertin, R.P., 1997. Phys. Rev. B 56, 321.

Cao, G., Alexander, C.S., McCall, S., Crow, J.E., Guertin, R.P., 1999. Mater. Sci. Eng. B $63,76$.

Cao, G., Balicas, L., Song, W.H., Sun, Y.P., Xin, Y., Bondarenko, V.A., Brill, J.W., Parkin, S., Lin, X.N., 2003. Phys. Rev. B 68, 174409.

Ciancio, R., Börjesson, J., Pettersson, H., Fittipaldi, R., Zola, D., Polichetti, M., Vecchione, A., Kittaka, S., Maeno, Y., Pace, S., Olsson, E., 2009a. Phys. Rev. B 80, 054110.

Ciancio, R., Pettersson, H., Börjesson, J., Lopatin, S., Fittipaldi, R., Vecchione, A., Kittaka, S., Maeno, Y., Pace, S., Olsson, E., 2009b. Appl. Phys. Lett. 95, 142507.

Crawford, M., Harlow, R.L., Marshall, W., Li, Z., Cao, G., Lindstrom, R.L., Huang, Q., Lynn, J.W., 2002. Phys. Rev. B 65, 214412.

Eom, C.B., Cava, R.J., Fleming, R.M., Phillips, J.M., vanDover, R.B., Marshall, J.H., Hsu, J.W.P., Krajewski, J.J., Peck Jr., W.F., 1992. Science 258, 1766.

Fittipaldi, R., Vecchione, A., Fusanobori, S., Takizawa, K., Yaguchi, H., Hooper, J., Perry, R.S., Maeno, Y., 2004. J. Cryst. Growth 271, 152.

Fittipaldi, R., Vecchione, A., Ciancio, R., Pace, S., Cuoco, M., Stornaiuolo, D., Born, D., Tafuri, F., Olsson, E., Kittaka, S., Yaguchi, H., Maeno, Y., 2008. Europhys. Lett. 83, 27007.
Fittipaldi, R., Maeno, Y., Vecchione, A., 2009. J. Phys. Condens. Matter 21, 254211. Fujimoto, T., Mitsuru, M., Masahashi, N., Kaneko, T., 2000. IOP Conf. Ser. 167, 79.

Grossin, D., Henrist, C., Mathieu, J.-Ph., Meslin, S., Harnois, C., Noudem, J.-G., Cloots, R., Chateigner, D., 2006. Supercond. Sci. Technol. 19, 190.

Huang, Q., Lynn, J.W., Erwin, R.W., Jarupatrakorn, J., Cava, R.J., 1998. Phys. Rev. B 58 8515.

Kim, D., Zink, B.L., Hellman, F., McCall, S., Cao, G., Crow, J.E., 2003. Phys. Rev. B 67, 100406(R).

Klein, L., Dodge, J.S., Ahn, C.H., Reiner, J.W., Mieville, L., Geballe, T.H., Beasley, M.R. Kapitulnik, A., 1996a. J. Phys. Condens. Matter 8, 10111.

Klein, L., Dodge, J.S., Ahn, C.H., Snyder, G.J., Geballe, T.H., Beasley, M.R., Kapitulnik, A., 1996b. Phys. Rev Lett. 77, 2774.

Koblischka-Veneva, A., Koblischka, M.R., Ogasawara, K., Murakami, M., 2002. Cryst. Eng. 5, 265.

Lichtenberg, F., Catana, A., Mannhart, J., Schlom, D.G., 1992. Appl. Phys. Lett. 60, 1138.

Mackenzie, A.P., Maeno, Y., 2003. Rev. Mod. Phys. 75, 657.

Mazin, I.I., Singh, D.J., 1997. Phys. Rev. B 56, 2556.

Perry, R.S., Kitagawa, K., Grigera, S.A., Borzi, R.A., Mackenzie, A.P., Ishida, K., Maeno, Y., 2004. Phys. Rev. Lett. 92, 166602.

Ramìrez-Rico, J., de Arellano-Lòpez, A.R., Martìnez-Fernàndez, J., Pena, J.I., Larrea, A., 2008. J. Eur. Ceram. Soc. 28, 2681.

Shaked, H., Jorgensen, J.D., Chmaissem, O., Ikeda, S., Maeno, Y., 2000. J. Solid State Chem. 154, 361 